\title{
Integration and analysis of multi-type high-throughput data for biomolecular knowledge discovery
}

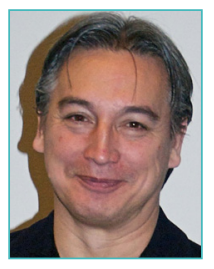

Erik Bongcam-Rudloff received his doctorate in medical sciences from Uppsala University, Sweden. He is now the Director of the SLU Global Bioinformatics Centre (SGBC) at the Swedish University of Agricultural Sciences.

Erik was the chairman of EMBnet (2003-2010), a science-based group of worldwide collaborating bioinformatics nodes. He also coordinated the "Test Cases" work package 4 in the FP6 EMBRACE project (2005-2010). The goal of WP4 was to collect test cases from the scientific community to identify real research problems and to provide solutions for content and tool integration. Bongcam-Rudloff is today Chair of SeqAhead, a Biomedical European COST Action: "Next Generation Sequencing Data Analysis Network" and Coordinator of ALLBIO, a FP7 project:
"Broadening the Bioinformatics Infrastructure to unicellular, animal, and plant science". He is also the founder of eBioinformatics.org the creators of eBiotools, eBioKit, eBioX and eBioKit.

His main research deals with development of bioinformatics solutions for the Life Sciences community.

In this talk he will discuss the new techniques that are now driving the generation of knowledge (especially in biomedicine and molecular life sciences) to new dimensions eg. NGS. He will also discuss the new opportunities in human and non-human research that these techniques create but also the new challenges in the design of ontologies for data and methods, and choosing common interoperability standards. 